\title{
Obesity, Metabolic Syndrome, and Cardiac Risk Factors: Going Gluten-Free, for Better or Worse?
}

\author{
Louise Emilsson $^{1,2,3}$ - Carol E. Semrad ${ }^{4}$
}

Published online: 21 June 2017

(C) Springer Science+Business Media, LLC 2017

The gluten-free diet (GFD) has gained in popularity with increased awareness of celiac disease and non-celiac gluten sensitivity. The reasons underlying its popularity in the general population are not well understood. Celebrity testimonials and its promotion as a weight control measure may contribute substantially to the "gluten-free lifestyle" in individuals with no evidence of celiac disease or gluten sensitivity.

Cardiovascular disease and the metabolic syndrome, major public health problems in the Western world, are largely affected by diet. Accordingly, it is of great importance to characterize the outcomes of any chosen diet on these problems. Celiac disease has been linked to a slightly increased risk of cardiovascular disease [1]. Several studies have reported increased blood pressure but lower body mass index (BMI), improved cholesterol profiles, and fewer smokers in individuals with celiac disease [2,3]. Mucosal healing in celiac disease is not associated with a decreased risk of myocardial infarction, stroke, or atrial fibrillation when compared to celiac individuals with persistent villous atrophy [4]. Hence, the impact of the GFD on metabolic and cardiovascular risk of celiac individuals

Louise Emilsson

lojsankik@hotmail.com

Carol E. Semrad

csemrad@medicine.bsd.uchicago.edu

1 Department of Epidemiology, Harvard T.H. Chan School of Public Health, Boston, MA, USA

2 Institute of Health and Society, University of Oslo, Oslo, Norway

3 Centre for Clinical Research and Vårdcentralen Värmlands Nysäter, County Council of Värmland, Karlstad, Sweden

4 University of Chicago Medicine, Chicago, IL, USA is not well characterized. High-quality data regarding the health effects of the GFD in non-celiac individuals are sparse, with no evidence supporting its benefit. On the contrary, high gluten intake has been reported to be associated with a lower risk of myocardial infarction [5].

In the current issue of Digestive Diseases and Sciences, Kim et al. [6] provide the first report addressing the risk of metabolic syndrome and cardiovascular disease in individuals without celiac disease following a GFD compared to individuals consuming dietary gluten. Using large-scale database analysis gathered by the National Health and Nutrition Examination Survey (NHANES) 2009-2014, the authors report a $1.3 \%$ prevalence of non-celiac individuals following a GFD in the general population [6]. Non-celiac GFD followers in the general population had a significant decrease in weight over 1 year, lower waist circumference, and higher HDL cholesterol levels. Nevertheless, there was no significant difference in the prevalence of metabolic syndrome or cardiac disease risk scores (smoking, hypertension, total plasma cholesterol) in those consuming a GFD compared to those eating gluten in the general population. The authors conclude that following a GFD is not associated with the most cardiovascular risk factors but is associated with female gender and superior weight reduction (lower BMI).

A cross-sectional study such as reported by Kim et al. unfortunately only allows for associations that cannot be directly translated into causal connections due to selection and other biases. As an example, consuming a GFD does not make one more female and hence might not contribute toward weight loss or to the lack of other metabolic or cardiac risk factors. As following a GFD is associated with higher educational attainment and income, and improved self-awareness [7], one might have predicted, a priori, that cardiovascular risk profiles would be superior to those of 
the average population eating gluten. Despite using a large database like NHANES, the study only identified 155 nonceliac GFD followers. Hence, the study lacks power to detect small differences in between groups. Since the response rates for the surveys used were $\sim 70 \%$, the data may not be representative of the whole population.

While this study provides insight into associations of a GFD with metabolic and cardiovascular risk, it also highlights the need for further research on the impact of the GFD in the general population. Millions in the USA and other countries already follow a GFD despite lack of indication. The important clinical question, how the GFD affects the risk of metabolic syndrome and cardiovascular disease in the general population, would ideally be answered in a prospective randomized controlled trial. Dietary trials are, however, notoriously difficult to conduct due to the multiple components included in a diet combined with difficulties with blinding, compliance, and other variables and potential biases. Hence, a more realistic approach to assess causality would include the conduct of well-characterized cohort studies, based on prospectively collected dietary data with long follow-up to study how the diet changes risk factors and cardiovascular outcomes over time. The current study by Kim et al. [6] is an important first step to frame the research question, to put the topic on the research agenda, and to increase awareness of the importance of research into the risks and benefits of the widespread use of the GFD.

To summarize, the GFD is a popular diet in the general population despite lack of evidence as to its beneficial or harmful effects. Kim et al. [6], using large database statistics, reported no significant difference in the prevalence of the metabolic syndrome or cardiovascular risk in the general population of GFD followers when compared to those eating gluten. There was a significant association with decreased weight in GFD followers that might provide a long-term health benefit if proven causal. The results need to be interpreted in the context of a retrospective cross-sectional survey with the most GFD followers being health conscious, well educated, and females, inserting conspicuous biases into the study design. Future prospectively collected cohort studies with well-defined research questions and robust methodologic designs or, optimally, randomized studies of the effect of the GFD on metabolic and cardiovascular risk are needed to answer its health impact.

\section{References}

1. Emilsson L, Lebwohl B, Sundstrom J, et al. Cardiovascular disease in patients with coeliac disease: a systematic review and metaanalysis. Dig Liver Dis. 2015;47:847-852.

2. Emilsson L, Carlsson R, Holmqvist M, et al. The characterisation and risk factors of ischaemic heart disease in patients with coeliac disease. Aliment Pharmacol Ther. 2013;37:905-914.

3. Stein AC, Liao C, Paski S, et al. Obesity and cardiovascular risk in adults with celiac disease. J Clin Gastroenterol. 2016;50:545-550.

4. Lebwohl B, Emilsson L, Frobert O, et al. Mucosal healing and the risk of ischemic heart disease or atrial fibrillation in patients with celiac disease; a population-based study. PLoS One. 2015;10:e0117529.

5. Lebwohl B, Cao Y, Zong G, et al. Long term gluten consumption in adults without celiac disease and risk of coronary heart disease: prospective cohort study. BMJ. 2017;357:j1892.

6. Kim H-S, Demyen MS, Mathew J, Kothari N, Feurdean M, Ahlawat SK. Obesity, metabolic syndrome and cardiovascular risk in gluten-free followers without celiac disease in the United States: results from the National Health and Nutrition Examination Survey 2009-2014. Dig Dis Sci. (Epub ahead of print). doi:10.1007/ s10620-017-4583-1.

7. Villafuerte-Galvez J, Vanga RR, Dennis $M$, et al. Factors governing long-term adherence to a gluten-free diet in adult patients with coeliac disease. Aliment Pharmacol Ther. 2015;42:753-760. 